•综述・

\title{
分子标记在濒危物种保护中的应用
}

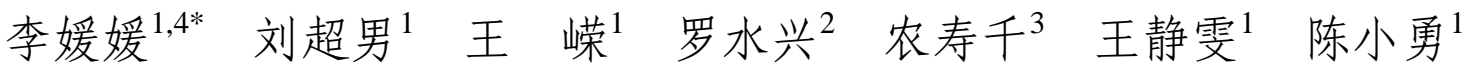

1 (华东师范大学生态与环境科学学院, 浙江天童森林生态系统国家野外科学观测研究站, 上海 200241)

2 (中国林业科学研究院热带林业研究所试验站, 海南乐东 572542)

3 (海南省林业科学研究所, 海口 571100)

4 (华东师范大学崇明生态研究院, 上海 200062)

摘要: 分子标记可揭示种群遗传和进化信息, 为制定濒危物种保护措施、指导恢复实践提供重要依据。本文主要 介绍了分子标记在濒危物种保护过程不同环节中的应用, 包括: (1)正确识别保护单元, 如排除隐存种和杂交种的 影响; (2)确定优先保护单元, 包括优先保护区域、优先保护物种、优先保护种群等; (3)指导迁地保护; (4)对保护工 作的动态监测和评估。文章最后探讨了分子标记应用于保护的发展方向, 如开展长期的种群遗传组成监测、切实 应用于保护管理实践、将基因组学等遗传信息用于全球变化背景下保护策略的制定等, 期望为分子标记技术在生 物多样性保护的研究和实践中提供参考。

关键词: 分子标记; 隐存种; 杂交; 进化显著单元; 管理单元; 迁地保护; 保护基因组学

\section{Applications of molecular markers in conserving endangered species}

Yuanyuan $\mathrm{Li}^{1,4^{*}}$, Chaonan $\mathrm{Liu}^{1}$, Rong Wang ${ }^{1}$, Shuixing $\mathrm{Luo}^{2}$, Shouqian Nong ${ }^{3}$, Jingwen Wang ${ }^{1}$, Xiaoyong Chen $^{1}$

1 Zhejiang Tiantong Forest Ecosystem National Observation and Research Station, School of Ecological and Environmental Sciences, East China Normal University, Shanghai 200241

2 Experimental Station of Research Institute of Tropical Forestry, Chinese Academy of Forestry, Ledong, Hainan 572542

3 Hainan Forestry Institute, Haikou 571100

4 Institute of Eco-Chongming, East China Normal University, Shanghai 200062

\begin{abstract}
Molecular markers have been widely applied in the conservation of endangered species for revealing their population genetic variation and evolutionary history, providing essential information for making conservation strategies and conducting restoration practices. Here, we introduced the applications of molecular markers at different stages of conservation efforts: (1) Molecular markers can be used to elevate the precision of identifying conservation units such as excluding the influence of cryptic species and hybridization. (2) Phylogenetic relationships are useful for distinguishing conservation priorities for biodiversity hotspots and discerning species and populations. (3) Molecular markers can contribute to instruct the strategies in ex situ conservation programs. (4) Dynamic monitoring and assessment of conservation activities can be achieved with molecular markers. Finally, we discussed the prospects of applying molecular markers for improving conservation strategies considering global change, including long-term monitoring of genetic dynamics, the use of conservation genetics in conservation management, and the development conservation genomics.
\end{abstract}

Key words: molecular markers; cryptic species; hybridization; evolutionary significant unit; management unit; ex situ conservation; conservation genomics

近一百多年来人口和经济的快速增长导致生 物多样性受威胁状况不断加剧, 人们对生物多样性
的保护也日益重视, 并取得了一些成效, 然而, 生 物多样性衰退的总体趋势仍没有得到根本改善

收稿日期: 2019-12-30; 接受日期: 2020-02-20

基金项目: 国家重点研发计划(2016YFC0503100)“极小种群野生植物种质资源保护技术研究与示范”课题(2016YFC0503102)和国家自然科学基金 (31670540)

* 通讯作者 Author for correspondence. E-mail: yyli@des.ecnu.edu.cn 
(Butchart et al, 2010)。究其原因，除了保护工作没有 切实落实外, 保育措施不当也是一个重要方面, 而 这主要是由于对物种的种群遗传特征缺少足够认 识导致的。分子标记是能反映个体、种群、物种或 更高层次遗传变异的核酸或蛋白质, 可为了解物种 种群生物学特征提供许多重要信息, 如遗传多样 性、基因流、交配系统、有效种群大小等, 有助于 预测物种应对快速变化环境的适应潜力。我国已有 一些综述介绍种群遗传学原理在生物多样性保护 中的应用 (李昂和葛颂, 2002; 王峥峰和彭少麟, 2003; 黄磊和王义权, 2004; 王峥峰和葛学军, 2009), 也有一些关于分子标记的介绍(张军丽等, 2000; 间 文德和乌云塔娜, 2006; 周伟和王红, 2014), 但未见 从物种的保护过程进行的阐述。近年来, 分子标记 技术在保护工作中的应用发展迅速, 并且在保护的 不同环节发挥的作用也不同。本文针对濒危物种保 护过程中的几个重要环节, 即从最初的识别保护单 元, 到确定就地保护的优先性, 再到指导迁地保护 的材料收集, 最后评价保护的效果等(图1), 介绍分 子标记在一系列保护环节中应用实践的新进展、新 思路。

\section{1 保护单元的识别}

确定正确的保护对象是生物多样性保护的前 提和基础。然而隐存种(cryptic species)的存在会引 起两个或多个物种的混淆, 导致目标物种识别困难, 种群大小被错误估算, 低估了物种的濒危程度, 导 致其在保护中被忽略而加剧灭绝速度; 或者是由于
种间杂交，导致种群规模小的稀有种被遗传同化甚 至灭绝，未能得到切实的保护。没有准确地识别隐 存种或杂交种，都会造成有限资金不能用在最需要 保护的物种上。

\section{1 隐存种的识别}

隐存种是指在形态上没有明显差异，但遗传分 化已达到物种或以上程度的种类(Fišer et al，2018; Struck et al, 2018)。对隐存种的鉴别能大大增强人们 对生物多样性的认知，提高保护的有效性。DNA条 形码(DNA barcoding)的应用为快速鉴定分类单元 提供了方便，动物中线粒体细胞色素氧化酶第I亚 基(mitochondrial cytochrome oxidase subunit I, COI) 是最常用的DNA条形码，而在植物中则常用叶绿体 核酮糖1,5-二磷酸羧化酶/加氧酶(ribulose-1,5-bisphosphate carboxylase/oxygenase, $r b c L$ )和成熟酶 $\mathrm{K}$ 蛋 白(maturase $\mathrm{K}, \mathrm{matK}$ )等基因片段的组合作为条形码, 通过条形码DNA序列的差异可以鉴定不同的分类 单元。例如, 中国大鲵(Andrias davidianus)是极危种 (critically endangered species)，不同种群间没有明 显的形态差异，长期以来被认为同属一个物种。最 近，Yan等(2018)采用线粒体COI、细胞色素b (cytochrome $b, C y t b$ )和置换环 (displacement-loop, D-loop)等DNA片段分析，发现该种由5个不同的支 系构成，每个支系可视为 1 个隐存种，且隐存种的 地理分布与水系有关。有时，研究人员会将分子方 法与形态、行为、生态、地理等特征综合起来鉴定 隐存种。铁青树科青皮木属(Quinchamalium)智利青 皮木 $(Q$. chilense) 在南安第斯山脉的常绿灌从、沙漠
保护过程

Conservation processes

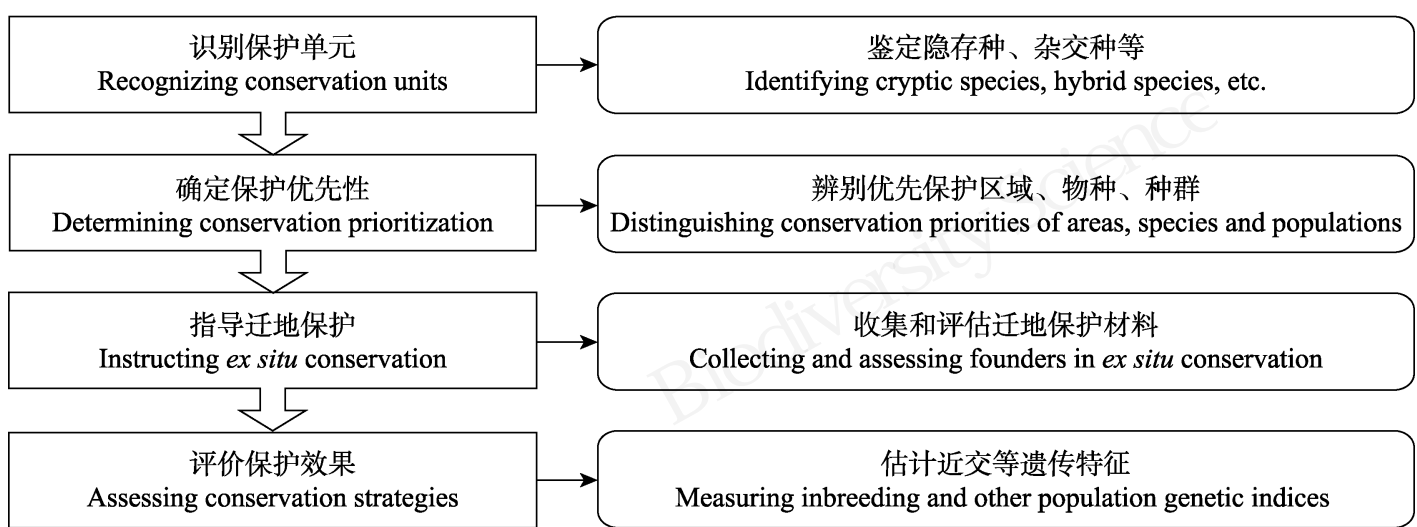

图1 分子标记在保护过程中的应用框架

Fig. 1 The framework for the applications of molecular markers in the conservation processes 
和高山温带森林生境中都有分布, 前两类生境中的 个体叶片宽、具短花柱花(thrum flowers); 而高山森 林生境中个体的叶片窄, 为长花柱花(pin flowers)。 研究者采用核糖体转录间隔区 (ribosomal DNA internal transcribed spacer, ITS)和叶绿体编码tRNA 的 $t r n L-F$ 区的条形码序列, 将高山森林生境的种类 区分出来, 但无法区分常绿灌从和沙漠区域中的个 体, 因此, 综合生态、形态和分子证据可认为该种 存在 2 个隐存种, 即常绿灌丛-沙漠种和高山种 (Lopez Laphitz et al, 2018), 因此作者提出将二者分 开保护的建议。

如果不能正确鉴别出隐存种, 将低估物种多样 性。优先保护区域大多是根据物种丰富度和特有种 的数量确定的, 而隐存种的发现能揭示未知的特有 种, 增加评估区的物种多样性。例如, 原来认为在 斯里兰卡分布的树蛙科有18个物种, 它们是由适应 辐射形成的，采用线粒体12S和16S编码基因分析后， 物种数增加到 100 多种, 这一地区也被评估为蛙类 多样性的热点地区(Meegaskumbura et al, 2002)。未 能识别出隐存种更严重的后果是高估了受胁物种 的个体数量, 从而可能低估其濒危程度。在原本数 量稀少的濒危物种中鉴定出隐存种, 意味着每个新 物种比之前预测的数量更少、分布范围更窄, 物种 的濒危程度可能更高, 并且不同物种可能需要不同 的保护策略。例如, Ravaoarimanana等(2004)采用线 粒体D-loop和Cytb片段分析发现, 马达加斯加岛一 种狐猴Lepilemur septentrionalis存在 2 个隐存种, 它 们可能是由于染色体重排而形成的, 其中北部 Sahafary种群是以前从未描述过的濒危种, 亟需单 独制定保护方案。

与之相反的是, 不同个体在遗传上属于同一个 物种, 但形态上表现出明显的差别, 若未正确鉴定 这种情形, 也不利于生物多样性保护。例如一些寄 生性桡足类(copepods)在形态上差异很大, 然而在 DNA条形码上没有明显分化, 因此它们仍属于同一 物种(Anton et al, 2016)。若因为形态不同而作为不 同的物种分别加以保护, 不仅可能降低种群间的基 因流，也将浪费珍贵的保护资源。

\section{2 杂交种的识别}

杂交种是遗传上相对独立的类群间交配形成 的, 对其保护地位存在较大的争议。虽然杂交对生 物多样性的维持有一定益处, 如可通过遗传拯救使
近交小种群避免绝灭, 但杂交也可能对生物多样性 产生负面影响, 比如打破物种间生殖障碍、融合独 特的进化支系、带来远交衰退等。若杂交发生在稀 有种(特别是极小种群物种)及其常见近缘种之间, 可能会因种群淹没(demographic swamping)、遗传同 化(genetic assimilation)引起种群规模小的稀有种灭 绝(Levin et al, 1996; Todesco et al, 2016)。例如, 野 外仅存于美国得克萨斯州和路易斯安那州沿海交 界地区的红狼(Canis rufus)由于种群规模严重下降, 导致与附近的郊狼(C. latrans)杂交严重, 许多后代 存在基因混杂的现象。为阻止红狼种群遗传淹没的 进一步发生, 美国鱼类及野生动植物管理局(United States Fish and Wildlife Service)开展了人为捕获野 生个体的行动以清除野外种群, 再通过野外捕获鉴定纯种-释放回归项目, 来保护红狼(Hinton et al, 2013)。Chiang等(2013)采用扩增片段长度多态性 (amplified fragment length polymorphism, AFLP)标 记研究显示, 台湾本地濒危种台东苏铁(Cycas taitungensis) 存在被园艺栽培的外来近缘种苏铁 (C. revoluta) 基因渗透的现象, 提示对本地种的保护需 考虑外来基因渐渗问题。

对于杂交种是否值得保护的问题, 目前国际上 多个保护组织也一直摇摆不定。许多学者认为杂交 种不能算作独立的物种, 无需担心它们是否灭绝, 只有非杂交种才能列在保护名单中(Allendorf et al, 2004)。许多组织认可这一观点, 如美国濒危物种法 案(Endangered Species Act)明确表示杂交种不值得 保护, “纯物种”被列入更高的保护级别中。比如, 有 的美洲豹(Felis concolor coryi)因是亚种间杂交而来, 被提议从保护名录中去除。然而, 由于生物多样性 保护和物种概念之间的争论, 1990年又废除了不保 护杂交种的相关法案, 目前美洲豹还列在保护名单 中(Rhymer \& Simberloff, 1996; Mallet, 2005)。Jackiw 等(2015)提出从生态(在遗传进化、适合度、生态系 统中的功能等方面是否有益)和伦理道德(在社会和 经济方面是否合乎道德)两方面综合考量, 对杂交 种开展不同管理策略的保护框架。

隐存种和杂交种的研究有利于科学地开展保护 工作, 制定有效的保护方案。但是, 我国隐存种研 究结果尚未纳入到区域生物多样性的评估中(徐伟 和车静, 2019), 鉴定的隐存种也未应用于保护方案 的制定中; 对于濒危物种的杂交种是否需要保护也 
鲜有考虑(商辉和严岳鸿, 2017)。因此, 在我国未来 需加强保护单元的鉴定与保护实践相结合。

\section{确定保护优先性}

\section{1 确定优先保护区域}

生物多样性热点地区是生态系统保护首要考 虑的区域, 力求用最低投入涵盖最高的生物多样性 (Pacicco et al, 2018)。目前对优先保护区的划分大多 只考虑物种的丰富度和特有性, 以及生态系统多样 性和脆弱性等方面, 而较少涉及遗传多样性; 据报 道，有关保护区的植物、节肢动物和鸟类的研究中 只有 6\%调查了遗传多样性(Muenchow et al, 2018)。 然而，物种多样性高的区域不一定是最优的保护地， 因为有可能物种组成相似而使保护的进化支系偏 向单一, 所以还需考虑物种的进化历史和生态功能 (Pollock et al, 2017), 而使用分子标记构建的系统发 育树可以提供类群的进化支系信息。Tucker等(2012) 采用线粒体、叶绿体和核DNA共30个基因序列对南 非好望角植物保护区(Cape Floristic Region)的山龙 眼科物种多样性进行评价, 分别计算了物种丰富 度、系统发育多样性(最大似然树特定分支所有支长 之和)、生物地理加权的进化独特性(一定范围内的 物种多度加权的系统发育多样性)等3个指标后发现, 生物地理加权的进化独特性能更好地反映南部地 区保护的优先性, 主要是因为这一地区进化的独特 性与地理上的稀有性呈正相关, 即稀有种具有更高 的进化独特性, 因此在确立优先保护区域时要特别 考虑这些稀有且独特的物种。

\section{2 确定优先保护物种}

除鉴别生物多样性保护区域的优先性外, 物种 保护也应考虑优先次序。IUCN对物种濒危程度的 评价主要依据分布区大小、种群数量、个体多少、 衰退速率、生存力分析等方面的标准(IUCN, 2012)。 然而, 不是所有物种都具有同等的系统发育地位, 某些物种有显著的进化贡献, 它们一旦灭绝意味着 重要的进化信息将丢失。Vane-Wright等(1991)介绍 了考虑物种进化独特性的方法, 通过物种在进化树 上与其他物种的分离程度检测进化独特性, 独特性 高的物种可作为优先保护 (phylogenetic conservation prioritization)对象。参照Isaac等(2007)提出的进化独 特及全局濒危(evolutionarily distinct and globally endangered, EDGE)方法, Ito等(2018)采用核ITS以及
叶绿体的 $r b c L 、 m a t K 、 R N A$ 聚合酶 $\beta$ 亚基基因(RNA polymerase beta subunit gene, $r p o B$ )和 $\beta^{\prime}$ 亚基基因 (RNA polymerase beta subunit gene, rpoC1)等5个片 段, 构建了水鳖科茨藻属(Najas) 21个物种的系统 进化树，计算了每个物种的EDGE值，该值由物种 进化独特性(物种从进化树末端至树根的每个节点 的分支长度除以该节点包含的物种数量的总和)与 灭绝风险(根据IUCN濒危等级赋值，等级越高赋值 越大)计算得出, 结果表明研究目标种弯果茨藻 $(N$. ancistrocarpa)具有明显的系统发育独特性，可作为 该属中第二位优先保护的物种。

\section{3 确定优先保护种群}

对于确定的保护物种，往往包含多个种群，鉴 于不同保护阶段和资金的限制，需要在受保护物种 中篎选出优先保护的种群。确定优先保护种群的依 据是遗传多样性及其分布, 这可以利用分子标记进 行种群遗传分析获得。可用的分子标记包括显性的 随机扩增多态性DNA (randomly amplified polymorphic DNA, RAPD)和AFLP，共显性的等位酶、限制 性片段长度多态性(restriction fragment length polymorphism，RFLP)、简单重复序列(simple sequence repeat, SSR; 又称微卫星(microsatellite)或短串联重 复序列(short tandem repeat, STR)), 还有基于DNA 序列信息的单核苷酸多态性(single nucleotide polymorphism, SNP)、应用高通量测序技术的简化基因 组测序(reduced representation genome sequencing, RRGS)、甚至基于基因组全序列的重测序等标记。 一般以遗传多样性(如期望杂合度或多态位点百分 比等)指标的高低考虑种群的优先保护顺序(Petit et al, 1998; 陈小勇等, 2002)。由于不同种群对环境响 应的潜力不同，保护重点应尽可能放在具最大进化 潜力的种群, 由此Petit等(1998)提出将通过稀化 (rarefaction)处理的等位基因丰富度作为确定优先 保护种群的指标, 这一参数表示每个位点等位基因 的数目多少, 并且高度依赖于有效种群大小而不是 取样种群的大小, 通过该指标鉴定每个种群在总遗 传多样性和遗传分化上的相对贡献，以此鉴别出优 先保护种群。但是, 这一方法依然是一种基于单个 种群本身遗传独特性的衡量。陆慧萍等(2004)则考 虑到种群之间的遗传变异, 将其分配到各个种群, 提出了一种综合考虑种群内遗传变异和种群间遗 传差异的遗传贡献率计算方法。 
优先保护种群的考虑还可基于进化显著单元 (evolutionary significant units, ESUs), 这一概念最早 由Ryder (1986)提出, 指一组进化独特且有长期历 史的种群集合, 作为识别和保持遗传多样性的一组 重点保护单元而设。ESUs一般根据质体DNA序列 构建的系统发育特征来鉴定, 把进化单系(monophyly)的一组种群归作1个ESU (Moritz, 1994a)。但 是, ESUs的划分有时存在一个进化支系涉及地理范 围过大的问题, 令保护工作难以在具体的物种监测 和管理中实施, 另一概念一一管理单元(management units, MUs)很好地解决了这个问题。MUs将种群遗 传特征与种群统计 (demography)特征结合, 通过辨 别核基因或质体基因在基因频率上有显著差异的 单元, 有时再结合一些种群的关键数量特征(如年 龄结构、生存力、繁殖力、性比等)确定MUs (Moritz, 1994b), 该划分缩小了保护单元的地理范围, 易用 于保护实践(Moritz, 1994a)。不同于ESUs, MUs主要 基于等位基因频率而不考虑等位基因的系统发育 关系, 考虑当前而不是历史的种群结构, 适用于短期 而不是长期的管理计划(Douglas \& Brunner, 2002)。

\section{3 指导迁地保护}

迁地保护(ex situ conservation)是对就地保护(in situ conservation)的有效补充, 最终目标是建立可持 续生存种群并使其将来能够回归(reintroduction)到 野外生境中(Williams \& Hoffman, 2009)。可持续生 存种群要求具有一定的遗传变异量, 因此迁地保护 前需要评估候选源种群的遗传组成状况, 用以选择 迁地保护种群的奠基者(founder)。大尺度上，考虑 到局域适应及避免远交衰退, 迁地保护的个体应该 在一个管理单元内选择(Witzenberger \& Hochkirch, 2011)。小尺度上, 收集野生个体的具体原则是既要 含有尽可能多的遗传变异, 又要代表一个管理单元 内易于丢失的遗传支系或低频率的等位基因(Saura et al, 2008)。

为达到上述目标, 广为运用的方法是从多个种 群中采集样本作为迁地保护种群的奠基者, 这些奠 基个体间的亲缘关系尽可能小, 并且不要只限制在 个别遗传支系内，该方法因使等位基因频率有均匀 分布的趋势, 可有效避免等位基因丢失(Saura et al, 2008; Witzenberger \& Hochkirch, 2011)。与之相反, 另一种方法提倡要尽量保持原来野生种群的等位
基因频率。但这个观点存在两方面的问题：一是迁 地种群在人工抚育过程中会因适应性而改变遗传 组成，最后达不到与原来野生种群相似的基因频率; 另一个是自然种群中低频率的有害突变基因如果 在迁地种群中因为保护良好反而提高了频率，将对 种群带来相当不利的后果，也会影响到将来回归的 种群。通过计算机模拟上述两种方法的效果，结果 是第一种选择亲本最小共祖率(minimum coancestry) 的方法对保持后代种群的遗传多样性更有效，但第 二种方法在一定程度上也能使后代的遗传多样性 保持在可接受的水平内(Saura et al, 2008)。

动物迁地保护实践中广泛采用的措施是圈养 繁育(captive breeding)，该方法最初选取奠基者时 主要是通过家系记录(pedigree data)来判断亲缘关 系。但是，除了记录出错的原因外，还不可避免地 假设奠基者间没有血缘关系，即奠基者假设(founder assumption), 这就低估了种群的近交系数, 错误 地估计后代的亲缘关系，可能导致做出错误的管理 决策(Russello \& Amato, 2004)。随着分子生物学技 术的发展, 采用分子标记指导圈养繁育计划取得了 良好效果, 近十几年来动物迁地保护研究采用的分 子标记由原来的等位酶、AFLP等转变为线粒体 DNA序列、SSRs以及基因组序列信息等。应用分子 标记, 短期内可以通过推断个体间亲缘关系, 建立 正确的配对亲本，提高繁育计划成功率; 长期来看, 通过计算不同支系的平均亲缘关系用于设计繁殖 配对方案，有利于保持种群遗传多样性，降低小种 群带来的遗传后果(Milián-García et al, 2015)。Witzenberger和Hochkirch (2011)分析了188篇文献认为, 与 50 个个体能够抵御近交衰退影响的 $50 / 500$ 法则 (Franklin, 1980)不同，最少100个迁地个体才能有效 抵御近交衰退和遗传多样性丧失。

对于植物来说, 种子库也是一种有效的迁地保 护方式，目前已有多个国家设立了种子库，如以色 列植物基因库(Israel Plant Gene Bank)、英国野生花 卉协会(New England Wild Flower Society)、我国西 南野生生物种质资源库(Germplasm Bank of Wild Species of Southwest China)等。采集种子时，要考虑 种内个体的空间分布，特别是对于种群间基因流低 的物种。Hoban和Schlarbaum (2014)应用计算机模拟 给出了迁地保护的采种方法：如有多个分布区域, 则在每个区域都采集一个种群(分散采样，dispersed 
sampling), 这样获得的等位基因数量是在一个分布 区域采集所有的种群(有限采样, constrained sampling)的1.75倍, 并且从这种广泛分布但数量不多的 种群中采集中等数量的个体(每种群25-30个)是最 优的策略。

\section{4 评估保护成效}

保护行动实施后有必要对种群遗传组成进行 监测和评价, 以便我们经常了解种群动态, 随时调 整保护策略。十几年来, 保护遗传学研究最理想的 分子标记是SSRs, 近年来利用高通量技术获得大规 模DNA序列也越来越多地应用于种群遗传研究。对 遗传数据进行常规的种群遗传分析可获得保护研 究的基础信息, 如观察杂合度、期望杂合度、近交 系数、有效种群大小等都是常用的指标。然后再通 过指标的状态推测导致遗传组成变化的原因, 评估 保护成效, 例如近交系数代表观察杂合度偏离哈迪 一温伯格平衡时期望杂合度的程度, 通过偏离情况 可以检测多种因素影响下的非随机交配现象, 如种 群结构(Wahlund效应)、近交程度等(Witzenberger \& Hochkirch, 2011)。

濒危物种的迁地保护和管理过程中, 近交是常 见的问题，但是有选择的杂交可能会引起远交衰退， 近交衰退和远交衰退分别是极度近缘和极度远缘 的 2 个亲本交配导致后代适合度较低的现象, 二者 给种群带来相似程度的风险(Edmands, 2007)。采用 SSRs等分子标记评价后，可以清晰地获知哪些种群 遭受过近交或远交衰退, 实际操作中可对近交衰退 的种群进行选择性杂交, 并至少在两个世代后就要 检测杂交效应, 以减小近交和/或远交衰退的风险 (Edmands, 2007)。

许多保护项目已经成功地开展回归行动, 濒危 物种的个体数量得到了成功恢复, 但是个体数量恢 复并不代表遗传变异已经成功恢复, 遗传变异是种 群长期存在的重要基础, 需要进行持续的监测。 White等(2018)调查了澳大利亚回归18年的4种动物 种群, 基于种群增长速度评估显示它们已经恢复成 功, 并且采用 SNPs 分子标记将回归种群与来源种 群的遗传特征进行比较, 回归种群的平均遗传多样 性接近奠基者种群的水平, 甚至有些回归种群由于 来源于遗传分化不大的几个种群, 通过这些个体的 相互交配还使回归种群的遗传多样性略微增大, 说
明保护中混合不同种群的个体可提高迁地种群的 遗传多样性。但是, 也不能一味地为提高遗传多样 性而混合，这会忽视恢复种群间的遗传差异，保持 一定的遗传独特性也是建立新种群需要考虑的。如 Li等(2005)采用RAPDs对比了水杉(Metasequoia glyptostroboides)人工种植种群和野生种群的遗传组成, 发现人工种群的遗传多样性低于野生种群, 并且遗 传组成比野生种群之间更相似，通过分子标记分析 不同年代建立的人工种群也显示存在很高的遗传 相似性(Chen et al, 2003), 这些结果表明水杉人工 种植种群的引种来源过于一致或由于频繁地无性 繁殖引种策略所致。

\section{结语}

分子标记应用于濒危物种和生态系统的保护 逐渐发挥了重要的作用, 尤其是目前对濒危物种的 生理、生态、所处环境条件等方面的了解已经越来 越深入，在保护策略中应考虑种群遗传因素。在未 来的生物多样性保护工作中，建议将分子标记技术 应用于以下几个方面，以更好地开展濒危物种的保 护和恢复。

(1)开展受保护物种遗传组成的长期变化监测。 目前这方面的研究非常少(Witzenberger \& Hochkirch, 2011), 遗传监测和种群动态监测一样, 是掌 握种群发展状况的良好评估手段, 种群遗传组成变 化依赖世代间的更替完成，因此更需要长期监测， 才能够反映种群的内在变化和进化。尤其是对于极 小种群野生植物而言，长期的遗传监测更具必要性 和可行性。

(2)将濒危物种种群遗传信息指导和应用于保 护管理实践。保护不仅要考虑种群统计学特征, 也 要在明确的遗传信息指导下完成配对、引种、迁地 保护、回归等实践项目，从而取得事半功倍的效果。 目前, 我国应用遗传信息开展保护实践在濒危动物, 尤其是重点保护动物如大熊猫(Ailuropoda melanoleuca)中较多, 主要应用于野生种群的复壮、圈养个 体亲子分析、圈养种群野外放归和再引入项目中, 极大地起到了维持种群的遗传多样性、避免近亲繁 殖、阻止种群衰退等作用(乔麦菊等, 2019)。在濒危 植物中，我国对部分物种的引种、回归项目已经考 虑到种群遗传方面，如保持遗传组成的多样性、避 免近交或远交、避免遗传淹没等(Ren et al, 2014), 
对保持濒危植物的适应性和可持续性起到积极作 用。但是, 目前多是从经验上或基于种群遗传信息 来实施的，鲜见根据单独个体的遗传信息实施的引 种、配对、回归等行动, 如若对极度濒危或极小种 群的植物考虑实施以个体遗传信息为基础的引种、 回归项目, 将使保护工作更加精准有效。

(3)将利用新出现的分子技术和分析方法提供 的遗传组成和适应性变异信息, 应用于全球变化背 景下保护策略的制定。基因组信息的不断积累为我 们成功实现保护目标提供了新的机会(Russello \& Amato, 2007), 保护遗传学(Conservation Genetics) 已经向保护基因组学(Conservation Genomics)发展。 我国作为“地球生物基因组计划”(Earth BioGenome Project) (Lewin et al, 2018)的主要成员之一, 相信随 着该计划的实施, 尤其是在优先测定受威胁物种基 因组的呼声下(Exposito-Alonso et al, 2020), 通过广 泛发掘序列变异(特别是适应性变异), 把基因组信 息应用于生物多样性保护将为全球变化(包括气候 变化、人类活动干扰等)背景下制定合适的保护措施 提供参考, 为更好地保护濒危物种和生态系统提供 有效的指导框架。

\section{参考文献}

Allendorf FW, Leary RF, Hitt NP, Knudsen KL, Lundquist LL, Spruell P (2004) Intercrosses and the U.S. Endangered Species Act: Should hybridized populations be included as westslope cutthroat trout? Conservation Biology, 18, 1203-1213.

Anton RF, Schories D, Wilson NG, Wolf M, Abad M, Schrodl M (2016) Host specificity versus plasticity: Testing the morphology-based taxonomy of the endoparasitic copepod family Splanchnotrophidae with COI barcoding. Journal of the Marine Biological Association of the United Kingdom, 98, 231-243.

Butchart SHM, Walpole M, Collen B, van Strien A, Scharlemann JPW, Almond REA, Baillie JEM, Bomhard B, Brown C, Bruno J, Carpenter KE, Carr GM, Chanson J, Chenery AM, Csirke J, Davidson NC, Dentener F, Foster M, Galli A, Galloway JN, Genovesi P, Gregory RD, Hockings M, Kapos V, Lamarque J, Leverington F, Loh J, McGeoch MA, McRae L, Minasyan A, Morcillo MH, Oldfield TEE, Pauly D, Quader S, Revenga C, Sauer JR, Skolnik B, Spear D, Stanwell-Smith D, Stuart SN, Symes A, Tierney M, Tyrrell TD, Vié J, Watson R (2010) Global biodiversity: Indicators of recent declines. Science, 328, 1164-1168.

Chen XY, Li YY, Wu TY, Zhang X, Lu HP (2003) Size-class differences in genetic structure of Metasequoia glyptostroboides Hu et Cheng (Taxodiaceae) plantations in Shanghai.
Silvae Genetica, 52, 3-4.

Chen XY, Lu HP, Shen L, Li YY (2002) Identifying populations for priority conservation of important species. Biodiversity Science, 10, 332-338. (in Chinese with English abstract) [陈小勇, 陆慧萍, 沈浪, 李媛媛 (2002) 重要物 种优先保护种群的确定. 生物多样性, 10, 332-338.]

Chiang YC, Huang BH, Chang CW, Wan YT, Lai SJ, Huang S, Liao PC (2013) Asymmetric introgression in the horticultural living fossil Cycas Sect. Asiorientales using a genome-wide scanning approach. International Journal of Molecular Sciences, 14, 8228-8251.

Douglas MR, Brunner PC (2002) Biodiversity of central alpine Coregonus (Salmoniformes): Impact of one-hundred years of management. Ecological Applications, 12, 154-172.

Edmands S (2007) Between a rock and a hard place: Evaluating the relative risks of inbreeding and outbreeding for conservation and management. Molecular Ecology, 16, 463-475.

Exposito-Alonso M, Drost H, Burbano HA, Weigel D (2020) The Earth BioGenome project: Opportunities and challenges for plant genomics and conservation. The Plant Journal, doi: 10.1111/tpj.14631.

Fišer C, Robinson CT, Malard F (2018) Cryptic species as a window into the paradigm shift of the species concept. Molecular Ecology, 27, 613-635.

Franklin IR (1980) Evolutionary change in small populations. In: Conservation Biology: An Evolutionary-Ecological Perspective (eds Soule ME, Wilcox BA), pp. 135-149. Sinauer, Sunderland.

Hinton JW, Chamberlain MJ, Rabon DR (2013) Red wolf (Canis rufus) recovery: A review with suggestions for future research. Animals, 3, 722-744.

Hoban S, Schlarbaum S (2014) Optimal sampling of seeds from plant populations for ex-situ conservation of genetic biodiversity, considering realistic population structure. Biological Conservation, 177, 90-99.

Huang L, Wang YQ (2004) The application of microsatellite DNA markers in conservation genetics of endangered animals. Biodiversity Science, 12, 528-533. (in Chinese with English abstract) [黄偣, 王义权 (2004) 微卫星分子标记 在濒危动物保护遗传学研究中的应用. 生物多样性, 12, 528-533.]

Isaac NJ, Turvey ST, Collen B, Waterman C, Baillie JEM (2007) Mammals on the EDGE: Conservation priorities based on threat and phylogeny. PLoS ONE, 2, e296.

Ito Y, Enju CY, Li J, Tanaka N (2018) Evolutionary distinctiveness and conservation priority of the endangered Najas ancistrocarpa (Hydrocharitaceae). Systematics and Biodiversity, 16, 45-54.

International Union for Conservation of Nature (IUCN) (2012) IUCN Red List Categories and Criteria: Version 3.1, 2nd edn. IUCN, Gland, Switzerland.

Jackiw RN, Mandil G, Hager HA (2015) A framework to guide the conservation of species hybrids based on ethical and eco- 
logical considerations. Conservation Biology, 29, 1040-1051.

Levin DA, Francisco-Ortega J, Jansen RK (1996) Hybridization and the extinction of rare plant species. Conservation Biology, 10, 10-16.

Lewin HA, Robinson GE, Kress WJ, Baker WJ, Coddington J, Crandall KA, Durbin R, Edwards SV, Forest F, Gilbert MTP, Goldstein MM, Grigoriev IV, Hackett KJ, Haussler D, Jarvis ED, Johnso WE, Patrinos A, Richards S, Castilla-Rubio JC, van Sluys M, Soltis PS, Xu X, Yang H, Zhang G (2018) Earth BioGenome Project: Sequencing life for the future of life. Proceedings of the National Academy of Sciences, USA, 115, 4325-4333.

Li A, Ge S (2002) Advances in plant conservation genetics. Biodiversity Science, 10, 61-71. (in Chinese with English abstract) [李昂, 葛颂 (2002) 植物保护遗传学研究进展. 生物多样性, 10, 61-71.]

Li YY, Chen XY, Zhang X, Wu TY, Lu HP, Cai YW (2005) Genetic differences between wild and artificial populations of Metasequoia glyptostroboides: Implications for species recovery. Conservation Biology, 19, 224-231.

Lu HP, Shen L, Zhang X, Fan XX, Chen XY (2004) Identifying populations for priority conservation. II. Models based on haplotype richness and their applications in Ginkgo biloba. Acta Ecologica Sinica, 24, 2312-2316. (in Chinese with English abstract) [陆慧萍, 沈浪, 张欣, 樊晓霞, 陈小 勇 (2004) 优先保护种群的确定. II. 单倍型丰富度模型 及在银杏中的应用. 生态学报, 24, 2312-2316.]

Lopez Laphitz RL, Ezcurra C, Vidal-Russell R (2018) Cryptic species in the Andean hemiparasite Quinchamalium chilense (Schoepfiaceae: Santalales). Systematics and Biodiversity, 16, 260-270.

Mallet J (2005) Hybridization as an invasion of the genome. Trends in Ecology and Evolution, 20, 229-237.

Meegaskumbura M, Bossuyt F, Pethiyagoda R, ManamendraArachchi K, Bahir M, Milinkovitch MC, Schneider CJ (2002) Sri Lanka: An amphibian hot spot. Science, 298, 379.

Milián-García Y, Jensen EL, Madsen J, Álvarez Alonso S, Serrano Rodríguez A, Espinosa López G, Russello MA (2015) Founded: Genetic reconstruction of lineage diversity and kinship informs ex situ conservation of Cuban Amazon parrots (Amazona leucocephala). Journal of Heredity, 106, 573-579.

Moritz C (1994a) Applications of mitochondrial DNA analysis in conservation: A critical review. Molecular Ecology, 3, 401-411.

Moritz C (1994b) Defining “evolutionary significant units” for conservation. Trends in Ecology and Evolution, 9, 373-375.

Muenchow J, Dieker P, Kluge J, Kessler M, von Wehrden H (2018) A review of ecological gradient research in the Tropics: Identifying research gaps, future directions, and conservation priorities. Biodiversity Conservation, 27, 273-285.

Pacicco L, Bodesmo M, Torricelli R, Negri V (2018) A methodological approach to identify agro-biodiversity hots- pots for priority in situ conservation of plant genetic resources. PLoS ONE, 13, e0197709.

Petit RJ, El Mousadik A, Pons O (1998) Identifying populations for conservation on the basis of genetic markers. Conservation Biology, 12, 844-855.

Pollock LJ, Thuiller W, Jetz W (2017) Large conservation gains possible for global biodiversity facets. Nature, 546, 141-144.

Qiao MJ, Ran JH, Zhang HM (2019) The application of microsatellite markers in giant panda research. Acta Theriologica Sinica, 39, 103-110. (in Chinese with English abstract) [乔 麦菊, 由江洪, 张和民 (2019) 微卫星标记在大熊猫研究 中的应用进展. 兽类学报, 39, 103-110.]

Ravaoarimanana IB, Tiedemann R, Montagnon D, Rumpler Y (2004) Molecular and cytogenetic evidence for cryptic speciation within a rare endemic Malagasy lemur, the northern sportive lemur (Lepilemur septentrionalis). Molecular Phylogenetics and Evolution, 31, 440-448.

Ren H, Jian SG, Liu HX, Zhang QM, Lu HF (2014) Advances in the reintroduction of rare and endangered wild plant species. Science China: Life Sciences, 57, 603-609.

Rhymer JM, Simberloff D (1996) Extinction by hybridization and introgression. Annual Review of Ecology and Systematics, 27, 83-109.

Russello MA, Amato G (2004) Ex situ population management in the absence of pedigree information. Molecular Ecology, 13, 2829-2840.

Russello MA, Amato G (2007) On the horns of a dilemma: Molecular approaches refine ex situ conservation in crisis. Molecular Ecology, 16, 2405-2406.

Ryder OA (1986) Species conservation and systematics: The dilemma of subspecies. Trends in Ecology and Evolution, 1, 9-10.

Saura M, Perez-Figueroa A, Fernandez J, Toro MA, Caballero A (2008) Preserving population allele frequencies in ex situ conservation programs. Conservation Biology, 22, 12771287.

Shang H, Yan YH (2017) Natural hybridization and biodiversity conservation. Biodiversity Science, 25, 683-688. (in Chinese with English abstract) [商辉, 严岳鸿 (2017) 自然 杂交与生物多样性保护. 生物多样性, 25, 683-688.]

Struck TH, Feder JL, Bendiksby M, Birkeland S, Cerca J, Gusarov VI, Kistenich S, Larsson K, Liow LH, Nowak MD, Stedje B, Bachmann L, Dimitrov D (2018) Finding evolutionary process hidden in cryptic species. Trends in Ecology and Evolution, 33, 153-163.

Todesco M, Pascual MA, Owens GL, Ostevik KL, Moyers BT, Hubner S, Heredia SM, Hahn MA, Caseys C, Bock DG, Rieseberg LH (2016) Hybridization and extinction. Evolutionary Applications, 9, 892-908.

Tucker CM, Cadotte MW, Jonathan Davies T, Rebelo TG (2012) Incorporating geographical and evolutionary rarity into conservation prioritization. Conservation Biology, 26, 593-601. 
Vane-Wright RI, Humphries CJ, Williams PH (1991) What to protect? Systematics and the agony of choice. Biological Conservation, 55, 235-254.

Wang ZF, Ge XJ (2009) Not only genetic diversity: Advances in plant conservation genetics. Biodiversity Science, 17, 330-339. (in Chinese with English abstract) [王峥峰, 葛学 军 (2009) 不仅仅是遗传多样性: 植物保护遗传学进展. 生物多样性, 17, 330-339.]

Wang ZF, Peng SL (2003) Plant conservation genetics. Acta Ecologica Sinica, 23, 158-172. (in Chinese with English abstract) [王峥峰, 彭少麟 (2003) 植物保护遗传学. 生态 学报, 23, 158-172.]

White LC, Moseby KE, Thomson VA, Donnellan SC, Austin JJ (2018) Long-term genetic consequences of mammal reintroductions into an Australian conservation reserve. Biological Conservation, 219, 1-11.

Williams SE, Hoffman EA (2009) Minimizing genetic adaption captive breeding programs: A review. Biological Conservation, 142, 2388-2400.

Witzenberger KA, Hochkirch A (2011) Ex situ conservation genetics: A review of molecular studies on the genetic consequences of captive breeding programmes for endangered animal species. Biodiversity and Conservation, 20, 18431861.

Xu W, Che J (2019) From cryptic species to biodiversity conservation in China: Status and prospects. Scientia Sinica
Vitae, 49, 519-530. (in Chinese with English abstract) [徐 伟, 车静 (2019) 从隐存种到我国生物多样性保护研究: 现状与展望. 中国科学: 生命科学, 49, 519-530.]

Yan F, Lü J, Zhang B, Yuan Z, Zhao H, Huang S, Wei G, Mi X, Zou D, Xu W, Chen S, Wang J, Xie F, Wu M, Xiao H, Liang Z, Jin J, Wu S, Xu CS, Tapley B, Turvey ST, Papenfuss TJ, Cunningham AA, Murphy RW, Zhang Y, Che J (2018) The Chinese giant salamander exemplifies the hidden extinction of cryptic species. Current Biology, 28, R590-R592.

Yan WD, Wuyun TN (2006) Methods of research on forest plant molecular ecology. Journal of Central South Forestry University, 26, 131-137. (in Chinese with English abstract) [间文德, 乌云塔娜 (2006) 森林植物分子生态学的研究 方法. 中南林学院学报, 26, 131-137.]

Zhang JL, Wang ZF, Li MG, Wang BS (2000) Molecular markers and their application in plant population research. Chinese Journal of Applied Ecology, 11, 631-636. (in Chinese with English abstract) [张军丽, 王峥峰, 李鸣光, 王伯荪 (2000) 植物种群研究中心的分子标记及其应用. 应用生态学报, 11, 631-636.]

Zhou W, Wang H (2014) Pollen dispersal analysis using DNA markers. Biodiversity Science, 22, 97-108. (in Chinese with English abstract) [周伟, 王红 (2014) 基于DNA分子标记 的花粉流动态分析. 生物多样性, 22, 97-108.]

(责任编委: 葛学军 责任编辑: 问文杰) 